\title{
Commercially available sun lamps and vitamin D formation
}

\author{
M. S. Devgun \\ Ph.D. \\ Alison J. M. Cruickshank \\ B. E. JOHNSON* \\ Ph.D. \\ C. R. PATERson \\ D.M., M.R.C.Path.
}

Departments of Biochemical Medicine, and *Dermatology, Ninewells Hospital, Dundee DDI 9SY

\begin{abstract}
Summary
Four commercially available, medium pressure mercury sun lamps were used to assess their effects on promoting vitamin D synthesis in the skin. It was found that all the lamps studied had vitamin D synthesizing spectral wavelengths and caused an increase in the serum concentrations of 25-hydroxyvitamin D. However, the ultraviolet and visible irradiance measurements showed that a considerable proportion of the ultraviolet radiation was below $290 \mathrm{~nm}$. It was not surprising, therefore, to find that these lamps caused adverse skin reactions. While a useful rise in vitamin $D$ production can be obtained with these sun lamps, the difficulty involved in avoiding skin reaction limits their usefulness. Such lamps are unlikely to provide a safe practical routine method for the prevention of vitamin $D$ deficiency in the home.
\end{abstract}

\section{Introduction}

Vitamin D is synthesized in the skin following exposure to ultraviolet radiation (UVR). Our understanding of the synthetic pathway, which starts with 7-dehydrocholesterol, has advanced recently with the demonstration of an intermediate compound, previtamin D (Havinga, 1973). While UVR is thought to be the major determining factor in the synthesis of vitamin D, other factors may be important (Boyle, 1980).

UVR has been used for the treatment of vitamin D deficiency and of skin disorders for many years (Bunker and Harris, 1937; van der Lugt and Rottier, 1958; Daniels, 1974). In parts of the Soviet Union, such as Siberia, so-called erythemal and sun-burn lamps are widely used in large industrial and instutional buildings including public schools (Zabaluyeva, 1958). Erythemal lamps are used

Requests for reprints to: Dr C. R. Paterson, Department of Biochemical Medicine, Ninewells Hospital, Dundee DD1 9SY. specifically for the purpose of correcting 'light starvation' which results in vitamin $D$ deficiency (Danzig, 1958; Karachevtseva and Groznaya, 1958).

In Britain, vitamin $\mathrm{D}$ deficiency can occur in house-bound or institutionalized elderly people (Chalmers et al., 1967) and in Asian immigrants (Gupta, Round and Stamp, 1974). Lack of UVR is a major contributory factor in both of these groups and one suggested method for the prevention of vitamin $\mathrm{D}$ deficiency is the provision of sources of UVR (Corless et al., 1975; Devgun et al., 1980).

Many types of UVR lamps are manufactured for cosmetic purposes (tanning) and 'health treatments'. It is well established that vitamin $D$ is synthesized by UVR of wavelengths between 280-320 nm (UV-B radiation) but, without due care, the use of lamps with emission in this range may result in acute sun-burn reactions. Over a long period premature ageing changes and skin cancer may occur and, unless the eyes are protected, cataracts may develop (World Health Organization, 1979). The purpose of this study was to assess the wavelength characteristics and the usefulness of some of the commonly available medium pressure mercury sun lamps in promoting vitamin D synthesis. It was hoped that the study would provide some insight into the value of UV-emitting lamps in the prevention of vitamin D deficiency in those at risk.

\section{Subjects and methods}

Twenty-five students, 8 males and 17 females, all aged between 20 and $22(21 \pm 0.7)$ years and all in good health, took part in this study. All subjects had similar life-style and a similar type of diet. The study was carried out between the end of January 1980 and the beginning of March 1980. These months were chosen because low levels of UV-B are present during this season in Dundee (latitude $56^{\circ} 30^{\prime}$ ). The subjects were randomly divided into experimental groups with a roughly equal number of students in 
each; one group of subjects was used as a control group.

Four sun lamps were used for the study (Table 1). Because these lamps were commercially available for use in the home, the 'skin-tanning' course treatment, as recommended by the manufacturers, was followed. However, for the Osram lamp it was, in most cases, impractical to organize the daily exposure recommended; although the ideal total exposure time was about $321 \mathrm{~min}$, the average obtained was $158 \mathrm{~min}$. All subjects wore protective eye goggles while exposed to UVR.

Blood samples were taken weekly from all 5 groups during the course of treatment and a followup sample was obtained a fortnight later. Serum samples were analysed for 25-hydroxyvitamin D (25-OHD) by a competitive protein binding technique (Preece et al., 1974). Serum calcium concentrations were measured on a Perkin-Elmer 303 atomic absorption spectrophotometer using a procedure based on the methods of Trudeau and Freier (1967) and Willis (1960). All subjects also filled in a questionnaire each day to indicate any adverse or erythematous skin reaction. For this study all the subjects were treated as though of normal skin sensitivity. Only the upper half, front and back, of the body was exposed to the lamps.

Spectral analyses of all 4 lamps were done using a spectro-radiometer (Spectro-Systems Ltd). Two methods were used in an attempt to determine the UV-B irradiance of the lamps. Firstly the total (UV and visible) irradiance was determined using a Hewlett Packard Flux-meter at a distance of $50 \mathrm{~cm}$, taking readings through $1 \mathrm{~cm}$ distilled water in a quartz cell to cut off wavelengths greater than $1000 \mathrm{~nm}$. Then a reading with, in addition, a WG 345 filter was taken for the determination of the output between 1000 and $320 \mathrm{~nm}$. By subtraction the UV-B output $(<320 \mathrm{~nm}$ ) could thus be measured. Secondly a 'sun-burn' meter was used to determine the UV counts/hr for wavelengths between 290 and $330 \mathrm{~nm}$. This method was used as previously de- $\varnothing$ scribed by Devgun et al. (1980). From these measure- $c$ ments a theoretical time required for minimal sun- $\overrightarrow{\vec{C}}$ burn with each lamp was calculated.

\section{Results}

Figure 1 shows the serum concentration of $25-\frac{\vec{\phi}}{\vec{\phi}}$ $\mathrm{OHD}$ in all 5 groups studied. After 3 weeks of

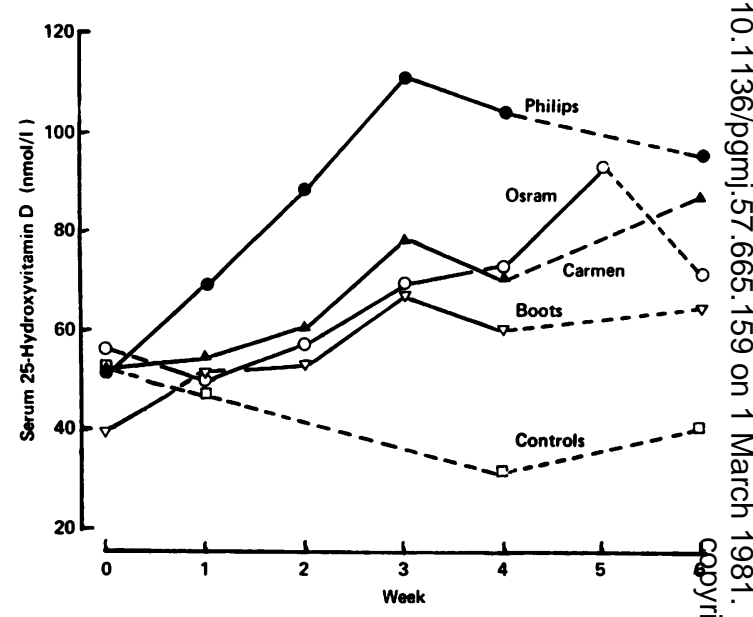

Fig. 1. Mean serum concentrations of 25-hydroxy- $\frac{0}{\partial}$ vitamin $D$ in UVR-exposed and control subjects. The unbroken lines indicate the period of exposure in each case, the broken lines indicate periods without exposure to UVR.

exposure, the serum concentrations of 25-OHD in $\stackrel{\circ}{3}$ the experimental groups were significantly higher $\supset$ than those in the control group $(P<0.001)$. The greatest rise was seen in subjects exposed to the Philips sun lamp. This group of subjects also showed 3 a significant rise in the serum concentrations of

TABLE 1. Details and the recommended instructions for the use of sun lamps

\begin{tabular}{|c|c|c|c|c|}
\hline & Osram & Boots & Philips & Carmen \\
\hline $\begin{array}{l}\text { Lamp } \\
\text { Watts (UV + IR) } \\
\text { Exposure distance (cm) }\end{array}$ & $\begin{array}{l}\text { Ultra-Vitalux } \\
300 \\
50\end{array}$ & $\begin{array}{c}\text { Combined UV and IR } \\
50+400 \\
50\end{array}$ & $\begin{array}{c}\text { Ultraphil HP.3114 UV and IR } \\
75+325 \\
75\end{array}$ & $\begin{array}{c}\text { Sundial } 128 \text { UV and IR } \\
50+200 \\
50\end{array}$ \\
\hline $\begin{array}{l}\text { Duration of initial } \\
\text { exposure (min) } \\
\text { Duration of final }\end{array}$ & $-0-2+0$ & 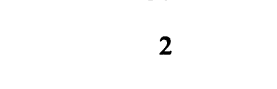 & 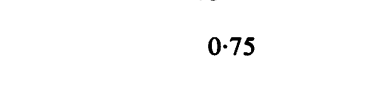 & 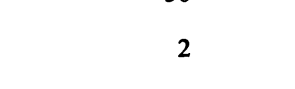 \\
\hline $\begin{array}{l}\text { exposure (min) } \\
\text { No. of sessions }\end{array}$ & $\begin{array}{c}15 \\
15-20\end{array}$ & $\begin{array}{c}2 \\
10 \\
\text { Alternate }\end{array}$ & $\begin{array}{c}5 \\
7 \\
\text { Alternate }\end{array}$ & $\begin{array}{c}15 \\
10\end{array}$ \\
\hline $\begin{array}{l}\text { Session frequency } \\
\text { Full course of treatment }\end{array}$ & Every day & Alternate days & Alternate days & Alternate days \\
\hline $\begin{array}{l}\text { (no. of days) } \\
\text { Total exposure time for }\end{array}$ & 25 & 19 & 13 & 19 \\
\hline a full course $(\mathrm{min})$ & 321 & 40 & 29.5 & 135 \\
\hline
\end{tabular}




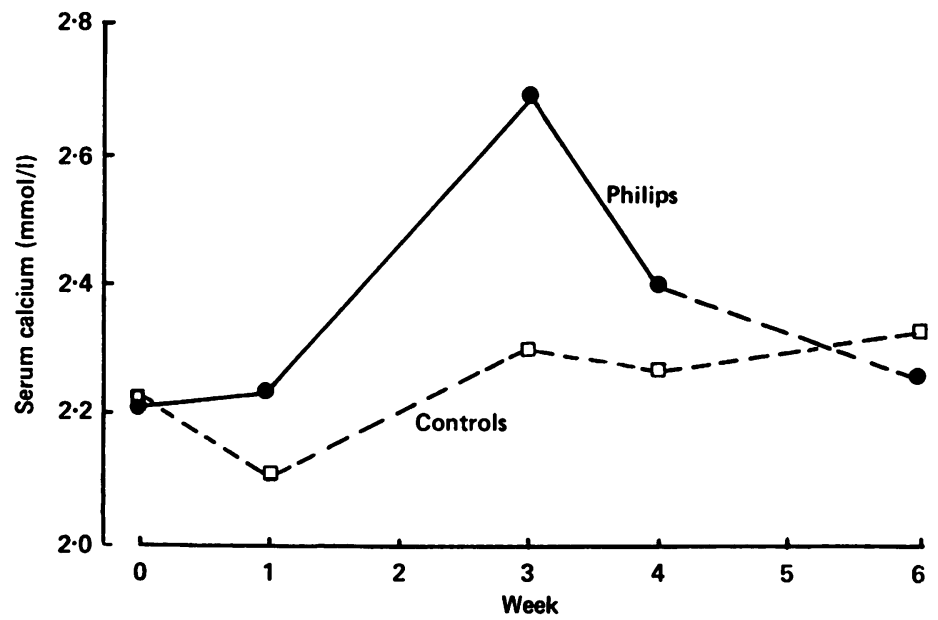

FIG. 2. Changes in serum calcium concentrations in control subjects and in subjects exposed to the Philips sun lamp.

calcium $(P<0.02)$ after 2 weeks of irradiation (Fig. $2)$; no change in the serum calcium was found in the other groups. All lamps caused a considerable rise in the serum concentrations of 25-OHD after 5 weeks of exposure.

There were no significant changes in the serum concentrations of 25-OHD in the follow-up samples in the experimental groups; the control group showed the expected seasonal decrease in January and February with a subsequent rise occurring in March $(P<0.01)$.

Table 2 shows the measurements made on the irradiance of the 4 lamps used in this study. It is apparent that Philips sun lamp has the highest levels of total and UV-B irradiance. This corresponds to its effects on serum 25-OHD and calcium concentrations. However, this lamp also caused an erythema after the second treatment in all of the 6 subjects in this group. The calculated minimal erythemal dose (MED) values are all very much higher than indicated by the manufacturers' instructions and erythema was obtained with lower exposure doses. With the Osram lamp erythema was seen in 3 out of the 5 subjects - in all these cases this was associated with a rise in serum 25-OHD concentrations. Similarly, 4 out of 5 subjects using the Carmen lamp produced an erythematous response which was also associated with a rise in plasma 25-OHD. Of all the 4 lamps used, only the Boots lamp did not appear to cause any adverse skin reactions, but the 25-OHD response was also comparatively modest.

Figure 3 shows the spectral scan of the Carmen sun lamp. Similar scans were observed for other sun

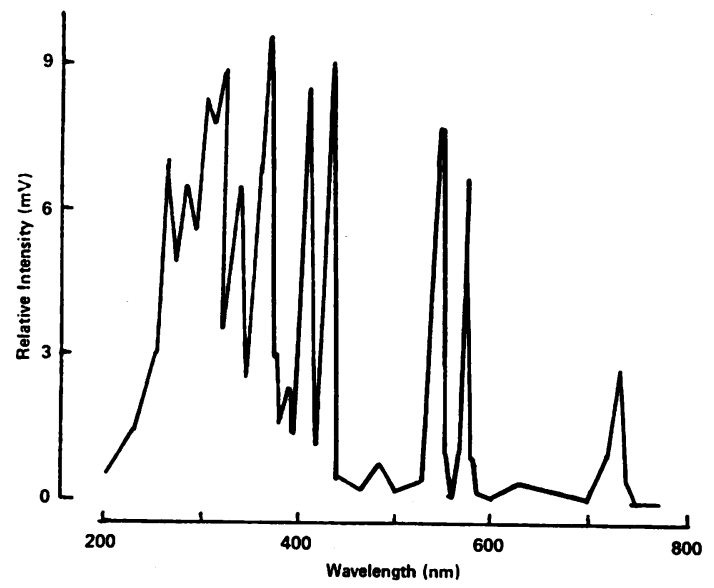

Fig. 3. Spectral scan of radiation output from Carmen sun lamp.

TABLE 2. Irradiance of the sun lamps and calculated minimal erythemal dose (MED)

\begin{tabular}{lccrr}
\hline & Osram & Boots & Philips & Carmen \\
\hline Total irradiance $\left(\mathrm{mW} / \mathrm{cm}^{2}\right)$ & 11.0 & 14.0 & 19.0 & 10.0 \\
UV B $\left(\mathrm{mW} / \mathrm{cm}^{2}\right)$ & 1.6 & 1.4 & 2.4 & 1.3 \\
$\begin{array}{l}\text { Calculated MED, using sun-burn } \\
\text { meter readings at a distance of }\end{array}$ & & & & \\
$50 \mathrm{~cm}(\mathrm{~min})$ & - & 13.0 & 3.5 & 10.0 \\
\hline
\end{tabular}


lamps. The spectral scans of Boots, Philips and Carmen sun lamps revealed that these lamps had appreciable irradiance below $280 \mathrm{~nm}$ wavelength. This was not found with the Osram sun lamp.

\section{Discussion}

The results show that exposure to commercially available home sun lamps with a dose schedule recommended by the manufacturers produced a significant increase in vitamin $\mathrm{D}$ synthesis in the skin as measured by the circulating concentrations of serum 25-OHD. The responses were similar in male and female subjects. Figure 1 shows that the initial increase in vitamin D synthesis was rapid and then 25-OHD concentrations reached a plateau. These findings are similar to those of Davie and Lawson (1979)

Exposure to the Philips sun lamp gave a significantly greater increase in serum 25-OHD concentrations $(P<0.05)$ than did other lamps, a result which would be expected with its higher UV-B output. No significant differences were obtained between the other lamps, again reflecting the UV-B irradiance measurements.

Only the subjects exposed to the Philips sun lamp had an increase in the serum concentration of calcium; this was seen after as little as $20 \mathrm{~min}$ of exposure. However, the serum calcium did not continue to rise despite continued exposure. This suggests that the immediate increase in plasma 25-OHD results in increased calcium absorption promoted by 1,25-dihydroxyvitamin $\mathrm{D}$; with the physiological homoeostatic mechanisms, these raised plasma levels of calcium are subsequently restored to normal. This homoeostatic control may be due to the removal of 25-OHD from plasma for storage or to a feedback inhibition of 1,25-dihydroxyvitamin D-production by the kidney. Feedback inhibition could also operate in the skin where 7-dehydrocholesterol is transformed into pre-vitamin D. Such a mechanism could explain the decrease of serum concentrations of 25-OHD after its initial rise at the start of the exposure.

Although the Philips sun lamp increased vitamin D synthesis, it also produced severe 'sun-burning' in all 6 subjects, even with the short exposure times recommended. Erythema also occurred with the other sun lamps with the exception of the Boots model which also produced the smallest rise in serum 25-OHD concentrations. It would therefore seem that, to obtain a greatly increased vitamin D synthesis in the skin, using home sun lamps, the exposure dose required is such as to produce a severe sunburn. Some increase in 25-OHD concentration may be obtained without adverse skin reactions as shown by the results with the Boots lamp. The authors feel, therefore, that the safe use of such lamps in the prevention of vitamin $\mathrm{D}-\frac{3}{\mathrm{Z}}$ deficiency must depend on precise adjustment of the $\varrho$ exposure dose, which would vary from lamp to lamp, and with different skin types. Even for $\overrightarrow{\overline{\bar{m}}}$ cosmetic purposes the value of these lamps is limitedby adverse skin reactions.

Calculations of the exposure times required to $\overline{\frac{}{5}}$ produce a minimal sun-burn reaction, based on the $\widehat{\widehat{ }}$ sun-burn meter counts, gave results which bear little $\stackrel{\varrho}{\nu}$ relationship to the UV-B irradiance measurements ${ }^{\infty}$ or to the MED values inferred from the manufac- $\overrightarrow{0}$ turers' recommendations. Examination of the spec- $\overrightarrow{-}$ tral scan shows that the emission for these lamps ${ }_{\sigma}^{\omega}$ contains relatively high amounts of radiation below$290 \mathrm{~nm}$ which is not detected by the sun-burn meter, $\frac{0}{3}$ but is very active in producing sun-burn. For lamps ir such as these, therefore, the sun-burn meter is not an. appropriate monitoring device.

\section{Acknowledgments}

This work was supported by a grant from the trustees of $O$ the Camilla Samuel Fund. We are very grateful to Boots the Chemists, House of Carmen Ltd and Philips Electrical Co. Ltd for providing the sun lamps and to Mr I. M. G. Kennedy for excellent technical assistance.

We thank all the medical students who took part in this $\stackrel{\rho}{\supset}$ study.

\section{References}

BOYLE, I.T. (1980) Vitamin D and ultraviolet radiation: Scottish Medical Journal, 25, 1.

Bunker, J.W.M. \& HARRIS, R.S. (1937) Precise evaluation of ultraviolet therapy in experimental rickets. New England Journal of Medicine, 216, 165.

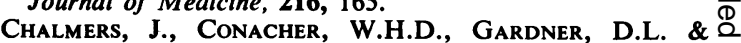
Scotr, P.J. (1967) Osteomalacia - a common disease in $\overrightarrow{\vec{B}}$ elderly women. Journal of Bone and Joint Surgery, 49B, 403.

Corless, D., Beer, M., Boucher, B.J., GuPta, S.P. \& COHEN, R.D. (1975) Vitamin D status in long-stay geriatric patients. Lancet, i, 1404.

Daniels, F. (1974) Physiological and pathological extra- 3 cutaneous effects of light on man and mammals, not mediated by pineal or other neuroendocrine mechanisms. In: Sunlight and Man-Normal and Abnormal PhotoBiologic Responses (Ed by Fitzpatrick, T.B., Pathak, M.A., 응 Harber, L.C., Seiji, M. \& Kukita, A.), p. 247. University of Tokyo Press, Tokyo.

DANZIG, N.M. (1958) Hygienic evaluation of the erythema lamp in treatment of ultraviolet insufficiency ('light $D$ starvation'). In: Ultraviolet Radiation, p. 199. Medgiz, 을. Moscow.

Davie, M. \& Lawson, D.E.M. (1979) Response of plasma $\widetilde{N}$ 25-OHD to standardized ultraviolet radiation. In: Vitamin D-Basic Research and its Clinical Application $ᄋ$ (Ed by Norman, A.W., Schaefer, K., Herrath, D., 心 Gregoleit, H., Coburn, J., Deluca, H., Mawer, E. \& Suda, T.), p. 163. Walter de Gruyter, Berlin.

Devgun, M.S., Paterson, C.R., Cohen, C. \& Johnson, B.E. (1980) Possible value of fluorescent lighting in the pre- $\mathbb{D}$ vention of vitamin D deficiency in the elderly. Age and Ageing, 9, 117.

GuPta, M.M., Round, J.M. \& Stamp, T.C.B. (1974) Spontaneous cure of vitamin $D$ deficiency in Asians during $\mathbb{D}$ summer in Britain. Lancet, i, 586. 
Havinga, E. (1973) Vitamin D-Example and challenge. Experientia, 29, 1181.

Karachevtseva, T.A. \& Groznaya, A.S. (1958) Effect of erythema lamp UVR on children afflicted with rheumatism. In: Ultraviolet Radiation, p. 174. Medgiz, Moscow.

Preece, M.A., O'Riordan, J.L.H., Lawson, D.E.M. \& KODICEK, E. (1974) A competitive protein binding assay for 25-hydroxy-cholecalciferol and 25-hydroxyergocalciferol in serum. Clinica chimica acta, 54, 235.

Trudeau, D.L. \& Freier, E.F. (1967) Determination of calcium in urine and serum by atomic absorption spectrophotometry. Clinical Chemistry, 13, 101.
VAN DER LugT, L. \& RotTier, P.B. (1958) Finsen therapy and vitamin D. Acta dermato-venereologica. Stockholm, 38, 264.

World Health Organization (1979) Ultraviolet Radiation: Environmental Health Criteria, 14, p. 1. W.H.O., Geneva.

WiLlis, J.B. (1960) The determination of metals in blood serum by atomic absorption spectroscopy. Spectrochimica acta, 16, 259.

ZabaluYeVA, A.P. (1958) Experimental incorporation of erythema lamps in indoor illumination systems of public schools. In: Ultraviolet Radiation, p. 249. Medgiz, Moscow. 